\title{
Erratum to: A Wald-type variance estimation for the nonparametric distribution estimators for doubly censored data
}

\section{Tomoyuki Sugimoto}

Published online: 16 April 2011

(C) The Institute of Statistical Mathematics, Tokyo 2011

\section{Erratum to: Ann Inst Stat Math DOI 10.1007/s10463-009-0251-3}

During the proof correction process, errors were introduced in the header of Table 1 by the typesetter. The correct version of the table is given here.

Table 1 Simulated averages of $\widehat{F}(\tau), n\left(\widehat{F}(\tau)-F^{*}(\tau)\right)^{2}, n \widehat{V}^{\text {Wald }}(\widehat{F}(\tau))$ and $n \widehat{V}^{\mathrm{LR}}(\widehat{F}(\tau))$ and simulated SDs of $n \widehat{V}^{\text {Wald }}(\widehat{F}(\tau))$ and $n \widehat{V}^{\mathrm{LR}}(\widehat{F}(\tau))$

\begin{tabular}{|c|c|c|c|c|c|c|c|}
\hline \multicolumn{8}{|c|}{ Outputs for censoring model I } \\
\hline \multirow[b]{2}{*}{$n$} & \multirow[b]{2}{*}{$\tau$} & \multicolumn{4}{|c|}{ Averages } & \multicolumn{2}{|l|}{ SDs } \\
\hline & & $\widehat{F}$ & $n\left(\widehat{F}-F^{*}\right)^{2}$ & $n \widehat{V}^{\text {Wald }}$ & $n \widehat{V}^{\mathrm{LR}}$ & $n \widehat{V}^{\mathrm{Wald}}$ & $n \widehat{V}^{\mathrm{LR}}$ \\
\hline \multirow[t]{9}{*}{100} & 0.1 & 0.0982 & 0.3575 & 0.1606 & 0.1559 & 0.0415 & 0.0419 \\
\hline & 0.2 & 0.2113 & 0.1879 & 0.1819 & 0.1772 & 0.0256 & 0.0261 \\
\hline & 0.3 & 0.3094 & 0.2108 & 0.2300 & 0.2261 & 0.0206 & 0.0213 \\
\hline & 0.4 & 0.4033 & 0.2787 & 0.2839 & 0.2813 & 0.0211 & 0.0217 \\
\hline & 0.5 & 0.5010 & 0.3173 & 0.3326 & 0.3314 & 0.0245 & 0.0246 \\
\hline & 0.6 & 0.6006 & 0.3541 & 0.3732 & 0.3731 & 0.0367 & 0.0364 \\
\hline & 0.7 & 0.7017 & 0.4462 & 0.4052 & 0.4063 & 0.0646 & 0.0642 \\
\hline & 0.8 & 0.8155 & 0.7873 & 0.4209 & 0.4228 & 0.1096 & 0.1091 \\
\hline & 0.9 & 0.9424 & 0.8001 & 0.4160 & 0.4185 & 0.1654 & 0.1652 \\
\hline
\end{tabular}

The online version of the original article can be found under doi:10.1007/s10463-009-0251-3.

T. Sugimoto $(\varangle)$

Department of Biomedical Statistics, Graduate School of Medicine,

Osaka University, 2-2 Yamada-oka, Suita, Osaka 565-0871, Japan

e-mail: sugimoto@medstat.med.osaka-u.ac.jp 
Table 1 continued

Outputs for censoring model I

\begin{tabular}{|c|c|c|c|c|c|c|c|}
\hline \multirow[b]{2}{*}{$n$} & \multirow[b]{2}{*}{$\tau$} & \multicolumn{4}{|c|}{ Averages } & \multicolumn{2}{|l|}{ SDs } \\
\hline & & $\widehat{F}$ & $n\left(\widehat{F}-F^{*}\right)^{2}$ & $n \widehat{V}^{\text {Wald }}$ & $n \widehat{V}^{\mathrm{LR}}$ & $n \widehat{V}^{\text {Wald }}$ & $n \widehat{V}^{\mathrm{LR}}$ \\
\hline \multirow[t]{9}{*}{400} & 0.1 & 0.1040 & 0.1529 & 0.1535 & 0.1489 & 0.0225 & 0.0227 \\
\hline & 0.2 & 0.2027 & 0.1678 & 0.1786 & 0.1739 & 0.0130 & 0.0132 \\
\hline & 0.3 & 0.3024 & 0.2158 & 0.2287 & 0.2248 & 0.0111 & 0.0114 \\
\hline & 0.4 & 0.4016 & 0.2639 & 0.2870 & 0.2844 & 0.0105 & 0.0108 \\
\hline & 0.5 & 0.5012 & 0.2956 & 0.3364 & 0.3352 & 0.0123 & 0.0124 \\
\hline & 0.6 & 0.6014 & 0.3476 & 0.3767 & 0.3768 & 0.0180 & 0.0178 \\
\hline & 0.7 & 0.7020 & 0.4008 & 0.4074 & 0.4086 & 0.0300 & 0.0297 \\
\hline & 0.8 & 0.8011 & 0.4400 & 0.4287 & 0.4307 & 0.0537 & 0.0535 \\
\hline & 0.9 & 0.9083 & 0.7216 & 0.4299 & 0.4324 & 0.1169 & 0.1168 \\
\hline \multirow[t]{9}{*}{900} & 0.1 & 0.1016 & 0.1461 & 0.1522 & 0.1477 & 0.0148 & 0.0149 \\
\hline & 0.2 & 0.2009 & 0.1748 & 0.1778 & 0.1730 & 0.0090 & 0.0092 \\
\hline & 0.3 & 0.3009 & 0.2298 & 0.2280 & 0.2241 & 0.0076 & 0.0078 \\
\hline & 0.4 & 0.4004 & 0.2807 & 0.2866 & 0.2841 & 0.0068 & 0.0070 \\
\hline & 0.5 & 0.5006 & 0.3268 & 0.3367 & 0.3355 & 0.0079 & 0.0079 \\
\hline & 0.6 & 0.6002 & 0.3820 & 0.3774 & 0.3774 & 0.0123 & 0.0122 \\
\hline & 0.7 & 0.7002 & 0.4215 & 0.4093 & 0.4105 & 0.0207 & 0.0206 \\
\hline & 0.8 & 0.8002 & 0.4425 & 0.4317 & 0.4338 & 0.0367 & 0.0366 \\
\hline & 0.9 & 0.9008 & 0.5033 & 0.4435 & 0.4454 & 0.0793 & 0.0794 \\
\hline \multirow[t]{9}{*}{1,600} & 0.1 & 0.1018 & 0.1562 & 0.1533 & 0.1488 & 0.0116 & 0.0117 \\
\hline & 0.2 & 0.2013 & 0.1783 & 0.1782 & 0.1735 & 0.0067 & 0.0069 \\
\hline & 0.3 & 0.3013 & 0.2256 & 0.2282 & 0.2242 & 0.0056 & 0.0058 \\
\hline & 0.4 & 0.4009 & 0.2852 & 0.2867 & 0.2841 & 0.0052 & 0.0053 \\
\hline & 0.5 & 0.5008 & 0.3453 & 0.3364 & 0.3352 & 0.0060 & 0.0060 \\
\hline & 0.6 & 0.6005 & 0.3885 & 0.3770 & 0.3771 & 0.0091 & 0.0090 \\
\hline & 0.7 & 0.6997 & 0.4189 & 0.4088 & 0.4100 & 0.0154 & 0.0153 \\
\hline & 0.8 & 0.7999 & 0.4446 & 0.4323 & 0.4344 & 0.0274 & 0.0273 \\
\hline & 0.9 & 0.9002 & 0.4678 & 0.4476 & 0.4499 & 0.0626 & 0.0625 \\
\hline
\end{tabular}

\begin{tabular}{|c|c|c|c|c|c|c|c|}
\hline \multirow[b]{2}{*}{$\imath$} & \multirow[b]{2}{*}{$\tau$} & \multicolumn{4}{|c|}{ Averages } & \multicolumn{2}{|l|}{ SDs } \\
\hline & & $\widehat{F}$ & $n\left(\widehat{F}-F^{*}\right)^{2}$ & $n \widehat{V}^{\text {Wald }}$ & $n \widehat{V}^{\mathrm{LR}}$ & $n \widehat{V}^{\text {Wald }}$ & $n \widehat{V}^{\mathrm{LR}}$ \\
\hline \multirow[t]{5}{*}{100} & 0.1 & 0.0648 & 0.7904 & 0.3374 & 0.3263 & 0.1126 & 0.1195 \\
\hline & 0.2 & 0.2053 & 0.5748 & 0.3182 & 0.3128 & 0.0604 & 0.0624 \\
\hline & 0.3 & 0.3107 & 0.3173 & 0.3169 & 0.3130 & 0.0377 & 0.0404 \\
\hline & 0.4 & 0.4084 & 0.3127 & 0.3200 & 0.3170 & 0.0259 & 0.0299 \\
\hline & 0.5 & 0.5025 & 0.2915 & 0.3221 & 0.3206 & 0.0215 & 0.0262 \\
\hline
\end{tabular}


Table 1 continued

\begin{tabular}{|c|c|c|c|c|c|c|c|}
\hline \multicolumn{8}{|c|}{ Outputs for censoring model II } \\
\hline \multirow[b]{2}{*}{$n$} & \multirow[b]{2}{*}{$\tau$} & \multicolumn{4}{|c|}{ Averages } & \multicolumn{2}{|l|}{ SDs } \\
\hline & & $\widehat{F}$ & $n\left(\widehat{F}-F^{*}\right)^{2}$ & $n \widehat{V}^{\text {Wald }}$ & $n \widehat{V}^{\mathrm{LR}}$ & $n \widehat{V}^{\text {Wald }}$ & $n \widehat{V}^{\mathrm{LR}}$ \\
\hline & 0.6 & 0.5986 & 0.3123 & 0.3207 & 0.3207 & 0.0260 & 0.0300 \\
\hline & 0.7 & 0.7003 & 0.3349 & 0.3171 & 0.3185 & 0.0404 & 0.0426 \\
\hline & 0.8 & 0.8111 & 0.5858 & 0.3149 & 0.3182 & 0.0674 & 0.0785 \\
\hline & 0.9 & 0.9434 & 0.7655 & 0.3269 & 0.3293 & 0.1107 & 0.1106 \\
\hline \multirow[t]{9}{*}{400} & 0.1 & 0.0969 & 0.8177 & 0.3569 & 0.3296 & 0.0894 & 0.1133 \\
\hline & 0.2 & 0.2050 & 0.3208 & 0.3199 & 0.3144 & 0.0330 & 0.0334 \\
\hline & 0.3 & 0.3037 & 0.3296 & 0.3193 & 0.3153 & 0.0188 & 0.0191 \\
\hline & 0.4 & 0.4030 & 0.3297 & 0.3222 & 0.3197 & 0.0126 & 0.0128 \\
\hline & 0.5 & 0.5021 & 0.3103 & 0.3235 & 0.3226 & 0.0106 & 0.0105 \\
\hline & 0.6 & 0.6017 & 0.2945 & 0.3217 & 0.3223 & 0.0127 & 0.0125 \\
\hline & 0.7 & 0.7013 & 0.3049 & 0.3173 & 0.3192 & 0.0189 & 0.0185 \\
\hline & 0.8 & 0.8003 & 0.3092 & 0.3165 & 0.3198 & 0.0328 & 0.0325 \\
\hline & 0.9 & 0.9081 & 0.6702 & 0.3542 & 0.3554 & 0.0958 & 0.0952 \\
\hline \multirow[t]{9}{*}{900} & 0.1 & 0.1031 & 0.4459 & 0.3632 & 0.3560 & 0.0625 & 0.0662 \\
\hline & 0.2 & 0.2021 & 0.3038 & 0.3179 & 0.3124 & 0.0223 & 0.0225 \\
\hline & 0.3 & 0.3018 & 0.3240 & 0.3184 & 0.3143 & 0.0130 & 0.0132 \\
\hline & 0.4 & 0.4011 & 0.3280 & 0.3222 & 0.3197 & 0.0085 & 0.0087 \\
\hline & 0.5 & 0.5012 & 0.3144 & 0.3240 & 0.3231 & 0.0068 & 0.0068 \\
\hline & 0.6 & 0.6007 & 0.3345 & 0.3223 & 0.3229 & 0.0083 & 0.0081 \\
\hline & 0.7 & 0.7001 & 0.3309 & 0.3185 & 0.3204 & 0.0132 & 0.0130 \\
\hline & 0.8 & 0.7998 & 0.3406 & 0.3183 & 0.3222 & 0.0230 & 0.0229 \\
\hline & 0.9 & 0.9010 & 0.4337 & 0.3649 & 0.3625 & 0.0642 & 0.0729 \\
\hline \multirow[t]{9}{*}{1,600} & 0.1 & 0.1023 & 0.3577 & 0.3638 & 0.3577 & 0.0438 & 0.0444 \\
\hline & 0.2 & 0.2012 & 0.2961 & 0.3181 & 0.3126 & 0.0162 & 0.0164 \\
\hline & 0.3 & 0.3010 & 0.3259 & 0.3185 & 0.3144 & 0.0099 & 0.0101 \\
\hline & 0.4 & 0.4009 & 0.3274 & 0.3262 & 0.3237 & 0.0065 & 0.0066 \\
\hline & 0.5 & 0.5010 & 0.3349 & 0.3237 & 0.3228 & 0.0050 & 0.0050 \\
\hline & 0.6 & 0.6008 & 0.3224 & 0.3223 & 0.3227 & 0.0064 & 0.0063 \\
\hline & 0.7 & 0.7002 & 0.3271 & 0.3184 & 0.3203 & 0.0098 & 0.0097 \\
\hline & 0.8 & 0.8004 & 0.3100 & 0.3185 & 0.3222 & 0.0170 & 0.0187 \\
\hline & 0.9 & 0.9008 & 0.3744 & 0.3688 & 0.3553 & 0.0488 & 0.0567 \\
\hline
\end{tabular}

Outputs for censoring model III

\begin{tabular}{|c|c|c|c|c|c|c|c|}
\hline \multirow[b]{2}{*}{$n$} & \multirow[b]{2}{*}{$\tau$} & \multicolumn{4}{|c|}{ Averages } & \multicolumn{2}{|l|}{ SDs } \\
\hline & & $\widehat{F}$ & $n\left(\widehat{F}-F^{*}\right)^{2}$ & $n \widehat{V}^{\text {Wald }}$ & $n \widehat{V}^{\mathrm{LR}}$ & $n \widehat{V}^{\text {Wald }}$ & $n \widehat{V}^{\mathrm{LR}}$ \\
\hline \multirow[t]{2}{*}{100} & 0.1 & 0.0841 & 0.7062 & 0.2998 & 0.2965 & 0.0898 & 0.0903 \\
\hline & 0.2 & 0.2114 & 0.4554 & 0.2915 & 0.2887 & 0.0515 & 0.0518 \\
\hline
\end{tabular}


Table 1 continued

Outputs for censoring model III

\begin{tabular}{|c|c|c|c|c|c|c|c|}
\hline \multirow[b]{2}{*}{$n$} & \multirow[b]{2}{*}{$\tau$} & \multicolumn{4}{|c|}{ Averages } & \multicolumn{2}{|l|}{ SDs } \\
\hline & & $\widehat{F}$ & $n\left(\widehat{F}-F^{*}\right)^{2}$ & $n \widehat{V}^{\text {Wald }}$ & $n \widehat{V}^{\mathrm{LR}}$ & $n \widehat{V}^{\text {Wald }}$ & $n \widehat{V}^{\mathrm{LR}}$ \\
\hline & 0.3 & 0.3125 & 0.2693 & 0.2833 & 0.2811 & 0.0282 & 0.0286 \\
\hline & 0.4 & 0.4066 & 0.2635 & 0.2712 & 0.2697 & 0.0148 & 0.0152 \\
\hline & 0.5 & 0.5023 & 0.2425 & 0.2654 & 0.2648 & 0.0086 & 0.0086 \\
\hline & 0.6 & 0.6015 & 0.2612 & 0.2704 & 0.2709 & 0.0158 & 0.0153 \\
\hline & 0.7 & 0.7014 & 0.2957 & 0.2806 & 0.2819 & 0.0318 & 0.0314 \\
\hline & 0.8 & 0.8065 & 0.4216 & 0.2857 & 0.2876 & 0.0573 & 0.0570 \\
\hline & 0.9 & 0.9335 & 0.6114 & 0.2828 & 0.2850 & 0.1128 & 0.1127 \\
\hline \multirow[t]{9}{*}{400} & 0.1 & 0.1055 & 0.4863 & 0.3023 & 0.2984 & 0.0610 & 0.0616 \\
\hline & 0.2 & 0.2060 & 0.2871 & 0.2947 & 0.2917 & 0.0283 & 0.0284 \\
\hline & 0.3 & 0.3040 & 0.2697 & 0.2852 & 0.2828 & 0.0151 & 0.0153 \\
\hline & 0.4 & 0.4026 & 0.2556 & 0.2733 & 0.2717 & 0.0074 & 0.0076 \\
\hline & 0.5 & 0.5015 & 0.2504 & 0.2670 & 0.2664 & 0.0036 & 0.0036 \\
\hline & 0.6 & 0.6009 & 0.2581 & 0.2731 & 0.2735 & 0.0077 & 0.0074 \\
\hline & 0.7 & 0.7005 & 0.2915 & 0.2843 & 0.2857 & 0.0156 & 0.0154 \\
\hline & 0.8 & 0.8001 & 0.2829 & 0.2929 & 0.2948 & 0.0300 & 0.0298 \\
\hline & 0.9 & 0.9032 & 0.4205 & 0.2928 & 0.2947 & 0.0676 & 0.0676 \\
\hline \multirow[t]{9}{*}{900} & 0.1 & 0.1040 & 0.2836 & 0.2980 & 0.2945 & 0.0414 & 0.0414 \\
\hline & 0.2 & 0.2023 & 0.2884 & 0.2927 & 0.2897 & 0.0189 & 0.0190 \\
\hline & 0.3 & 0.3015 & 0.2740 & 0.2847 & 0.2822 & 0.0099 & 0.0100 \\
\hline & 0.4 & 0.4008 & 0.2730 & 0.2735 & 0.2719 & 0.0049 & 0.0050 \\
\hline & 0.5 & 0.5007 & 0.2634 & 0.2678 & 0.2672 & 0.0024 & 0.0024 \\
\hline & 0.6 & 0.6004 & 0.2680 & 0.2740 & 0.2744 & 0.0050 & 0.0049 \\
\hline & 0.7 & 0.7001 & 0.2976 & 0.2854 & 0.2868 & 0.0106 & 0.0104 \\
\hline & 0.8 & 0.7998 & 0.2786 & 0.2936 & 0.2956 & 0.0202 & 0.0200 \\
\hline & 0.9 & 0.8999 & 0.3127 & 0.2975 & 0.2997 & 0.0453 & 0.0452 \\
\hline \multirow[t]{9}{*}{1,600} & 0.1 & 0.1035 & 0.3322 & 0.2992 & 0.2958 & 0.0322 & 0.0322 \\
\hline & 0.2 & 0.2018 & 0.2956 & 0.2934 & 0.2903 & 0.0142 & 0.0143 \\
\hline & 0.3 & 0.3011 & 0.2956 & 0.2954 & 0.2930 & 0.0077 & 0.0078 \\
\hline & 0.4 & 0.4011 & 0.2861 & 0.2838 & 0.2822 & 0.0038 & 0.0039 \\
\hline & 0.5 & 0.5010 & 0.2794 & 0.2777 & 0.2771 & 0.0018 & 0.0018 \\
\hline & 0.6 & 0.6009 & 0.2849 & 0.2838 & 0.2842 & 0.0039 & 0.0038 \\
\hline & 0.7 & 0.7003 & 0.2906 & 0.2902 & 0.2916 & 0.0079 & 0.0078 \\
\hline & 0.8 & 0.8008 & 0.2800 & 0.2933 & 0.2953 & 0.0144 & 0.0143 \\
\hline & 0.9 & 0.9011 & 0.2956 & 0.2957 & 0.2983 & 0.0315 & 0.0315 \\
\hline
\end{tabular}

